\section{Irren ist menschlich}

Am 25. Januar dieses Jahres fand in Bern eine von der FMH organisierte Tagung zum Thema "Risiken, Fehler und Patientensicherheit" statt. In dieser und in der nächsten Ausgabe der Schweizerischen Ärztezeitung veröffentlichen wir Referate, die an dieser Tagung gehalten wurden.

Die Tagung stiess damals auf lebhaftes Interesse, denn das Thema war aus aktuellem Anlass in aller Munde: Einzelfälle ärztlicher Behandlungsfehler hatten zuvor die Schlagzeilen bereichert, und manch ein Politiker witterte dabei gleich höchste Gefahr für die allgemeine Patientensicherheit und schlug Alarm. Zum Teil heftige Diskussionen gingen mit ebenso unvermeidbarem wie bedeutungslosem "battle smoke" einher. Dieser hat sich inzwischen verzogen. Geblieben ist, so will mir scheinen, die für ein gewisses Publikum doch verblüffende Erkenntnis, dass sich Ärztinnen und Ärzte seit jeher und mit steter Sorge um Patientensicherheit kümmern. Aufärztlicher Seite wurden, im Gegenzug, die Zeichen der Zeit erkannt: Wenn auch Ärztinnen und Ärzte wie nie zuvor ihren Patientinnen und Patienten tagtäglich ein beeindruckendes Mass an Sicherheit bieten können, so bergen gerade die Mittel, die diesen Fortschritt ermöglicht haben, auch das Potential, Schaden anzurichten - wie nie zuvor. Es stände daher einer Medizin, welche sich von paternalistischem Gehabe verabschieden möchte, schlecht an, den da und dort sich bietenden, fairen und ernsthaften Dialog mit Laien und Politikern zu verschmähen. Fehler in der Medizin sind - im noblen Sinn des Wortes zu Recht ein Politikum. Wenn Fehler bei der Behandlung eines Patienten passieren, sind alle betroffen: der Patient, seine Angehörigen, der behandelnde Arzt, das soziale und berufliche Umfeld, ja selbst die Gesellschaft; man brauche sich dabei nur vorrechnen zu lassen, wieviel durch Vermeiden ärztlicher Fehler eingespart werden könnte - Summen, die anderswo bessere Verwendung fänden.
Fehler sind, in der Medizin wie anderswo auch, vermeidbar. Lapidares Ergebnis langer Diskussionen. Sonst würde man ja auch nicht von Fehlern sprechen, sondern von Unfällen oder Einwirkungen höherer Gewalt. Solange aber keine Wunder geschehen, gelten die Naturgesetze; mit diesen indessen können wir immerhin Berechnungen anstellen. Anekdotisch, aber bedeutsam ist der kürzlich publizierte Vorsatz des renommierten British Journal of Medicine (BMJ), das Wort "accident" aus seinem Sprachgebrauch auszumerzen [1]: "An accident is often understood to be unpredictable - a chance occurrence or an 'act of God' - and therefore unavoidable. However, most injuries and their precipitating events are predictable and preventable. That is why the BMJ has decided to ban the word accident." Statt dessen sollen gelten: "injuries", "crashes", "overdoses" ... "errors".

Irren ist menschlich. Aus Fehlern kann und soll man lernen. Wenn Fehler eingestanden und mitgeteilt werden dürfen - keine Strafe in Sicht! -, können daraus für alle profitable Lehren gezogen werden. Einzelne fehlerhafte Individuen anzuprangern, ist wenig hilfreich, vielmehr müssen "Systeme», welche Fehler geschehen lassen, analysiert und verbessert werden. Diese und andere altbewährte Weisheiten werden nun neu entdeckt und erfreuen sich grosser Beliebtheit. Um so besser!

Wir dürfen gespannt sein, welche Lösungsansätze den Weg in die Praxis finden werden. Sicher können Erfahrungen aus anderen Ländern und aus anderen Branchen im Umgang mit "critical incidents" als wertvolle Modelle dienen. Es ist auf jeden Fall zu wünschen, dass auf diesem Gebiet in optimistischzupackender Art vorgegangen wird. Jeder grössere Fehler, der vermieden werden kann, ist eine individuelle Tragödie weniger.

Markus Trutmann

1 Davis RM, Pless B. BMJ bans "accidents". Br Med J 2001;322:1321-2. 